\title{
Cell Zooming for Energy Efficient Wireless Cellular Network
}

\author{
V. Prithiviraj ${ }^{1}$, S. B. Venkatraman ${ }^{2}$ and R. Vijayasarathi ${ }^{3}$ \\ ${ }^{1}$ Rajalakshmi Institute of Technology, Chennai. \\ ${ }^{2,3}$ Pondicherry Engineering College, Puducherry \\ ${ }^{1}$ profvpraj@gmail.com, ${ }^{2}$ venkat@pec.edu, ${ }^{3}$ r.vijayasarathi@pec.edu
}

Received: September 18, 2013; Accepted: September 27, 2013

\begin{abstract}
The dynamic growth of Information and Communication Technology (ICT) have considerably increased the energy requirements and also contribute a growing fraction to $\mathrm{CO}_{2}$ emissions, thereby creating a need for wireless communications to be more energy-efficient. In this paper, Cell Zooming concept is implemented for reducing the overall power consumption of the network for a five cell microcellular network adopting SON technique. When the traffic load is low in central base station, it could be switched off while its users are given coverage by cell zooming the neighboring cells. Simulation results indicate that by using the Cell Zooming concept, nearly $20 \%$ of the overall power consumption of the network can be reduced. This would herald the emergence of the Green Base Station in Wireless Cellular Network.
\end{abstract}

Keywords: ICT, energy-efficient, cell zooming, SON.

\section{Introduction}

There is an enormous rapid growth in wireless cellular networks due to the explosive increase in traffic volumes as a consequence of the exponential rise in subscriber numbers. Network providers are doing their best to fulfill user requirements economical. The cell size in a cellular network is said to be the area where mobile users can get the common control signals and data signals

Journal of Green Engineering, Vol. 3, 421-434.

doi: $10.13052 /$ jge 1904-4720.344

(c) 2013 River Publishers. All rights reserved. 
transmitted from the base station. Cell size and capacity are generally static at the phase of network planning, pertaining to the estimated value of peak traffic load. But, persuasive spatial and temporal fluctuations are always there due to the mobile nature of users and transmission of data in short sudden bursts [1].

Usually for a cellular network in the urban at daytime the traffic load is comparatively heavy in workplaces and light in housing areas and it is just the conflicting during the dusk. If the network capacities are specified pertaining to the peak traffic volume of every cells, load distribution will not be appropriate i.e., there are always some traffic load imbalances. Specific cells are under lighter load and some are heavy loaded. Because of this static cell deployments couldn't be an optimum solution as traffic loads experiences fluctuation.

Traffic load variations are further severe in succeeding generation cellular networks that is headed for smaller cells like micro, pico and femto-cell, making the network deployments more challenging.

The problem of traffic load fluctuations makes certain MUs incapable from getting service. For example, traffic loads become high in some cells over the scheduled capacity, making some MUs left without getting servicefrom that particular BS. In the same instant neighboring cells are left with lighter traffic load. Suitable load balancing schemes could be used to resolve this problem to certain extend [2].

In the exponentially growing world energy consumption is becoming a vital issue, also usage of energy sources are creating larger negative impact on the environment with global warming because of the carbon emission. For example, a standard $3 \mathrm{G}$ base station to produce $40 \mathrm{~W}$ of output RF power requires $500 \mathrm{~W}$ of input power, with 12,000 base stations it consumes more than 50GWh of input power every year in a network [3]. The carbon foot print from the base stations is also growing exponentially because of the diesel power generators that are used as backhaul for electrical grids. This evidently indicates the biggest power issue that is concerning cellular networks is the power consumed by base stations. This shows that "Green cellular network" deployment needs a primary focus on power consumption reduction in base stations.

Green Communications has to felicitate the global environment and help the telecommunication operators economically giving profitable and sustainable business. This is possible by finding a radio networking solution that could be energy-efficient and also resource-efficient without compromising the quality of service. 
In order to achieve green communication by saving power in base stations, green base stations have to be implemented. Some of solutions to green base stations are switching off base stations periodically by some power saving protocols, and improving the power amplifiers which is the maximum energy consumption part in base stations, using renewable energy resources, network self-organizing techniques ( $\mathrm{SON}$ ), etc. There are many base station switching on/off schemes are proposed in papers [4]-[7].

From the above solutions, SON is one among the promising areas for saving operational expenditures in the next-generation radio access networks like 3GPP-LTE. The Self-organizing networks has the ability to self-configure itself for balancing loads, can do optimizations and healings, cell outage managements and management of repeater and relays, etc.

SON can be established by switching off certain BSs which are under lighter traffic load (work places during night times) and provide considerable energy saving. This could be realized by the concept of cell zooming. Cell zooming of flexibly adjusting the technique is capable cell size with respect to traffic variations. With this adaptive nature, cell zooming is likely to balance the reduction of energy consumption and traffic load [8].

Cell zooming technique is realized by cell zooming in, to reduce the cell size while a cell is congested and cover only users present in the smaller area. Hence releases itself from the congestion while other users are provided coverage by cell zooming out the neighboring cells avoiding coverage hole. When a cell is less congested it can also be switched off and the users in the switched off cell's region is covered with cell zooming out the neighboring cells. This cell zooming is done by relaying, or by altering the transmission power of BS, else with BS cooperation [9].

In this paper, a scenario is introduced to illustrate the implementation of cell zooming concept and thereby it explains how the overall network energy consumption is reduced with the help of cell zooming technique.

\section{System Model}

The case investigated here is focused in wide area cellular network scenarios. The total network contains five same size cells, i.e., cell1, cell2, cell3, cell4 and cell5 each representing its Base Stations (BSs), i.e., BS1, BS2, BS3, BS4 and BS5, as presented in Fig. 1. Here each BS consists of six sectored antennas that are used to cover its geographic region. The BS transmission power for each user is calculated according to user's distance in the cell. Since these transmit power values are required for comparing with the minimum 


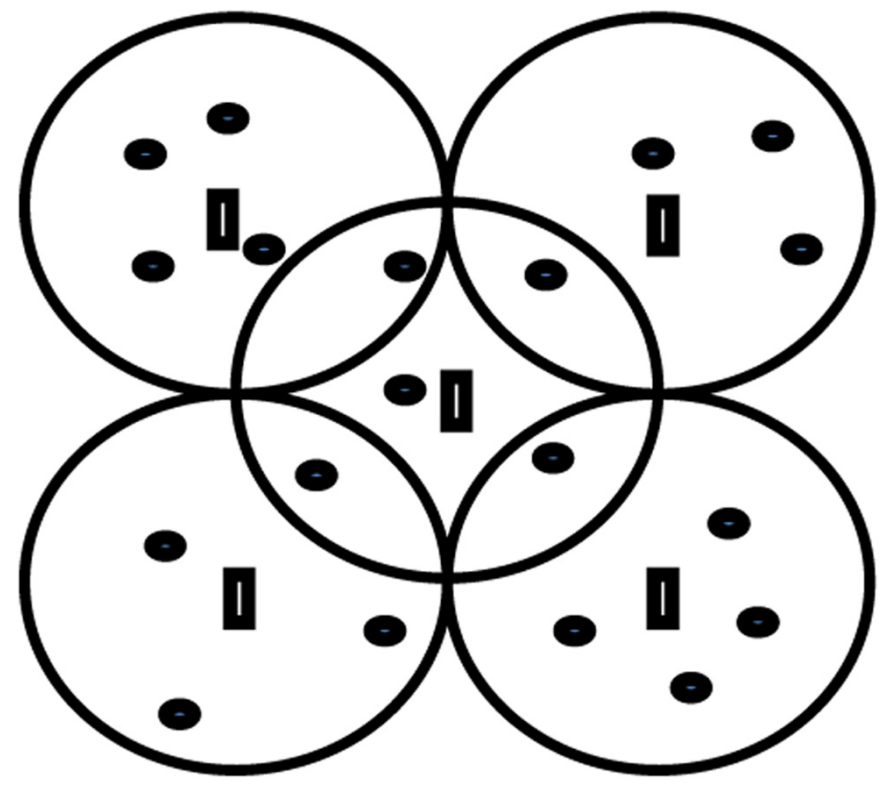

Figure 1 Cell with original size

receiver power of mobile station, thereby to keep up with signal to noise ratio (SNR) value.

In this paper, with respect to the presence of active MUs in the center cell (cell5), it is switched off. While the neighboring cells can increase their transmit power in any of the sectors according to the geographical region and cover the users in the switched off cell as in Fig. 2. In the same way, if the central cell is densely populated, some users may be left without covering due to scarcity of bandwidth and at the same time if the neighboring cells has less users, the neighboring cells can extend their coverage by cell zooming out and cover those users in central cell. In our scenario, cell zooming out by the neighboring base station to cover the users in the central switched off cell is analyzed.

The reasons for switching off the central cell is because its geographic region can be covered easily by increasing transmit power of the neighboring cells.

Distance between each of the users in the switched off cell and the center of the neighboring base stations is calculated. All the distance is sorted and the nearest neighboring cell extends its coverage by zooming out to give coverage for those users. In certain cases all the neighboring base stations may have 


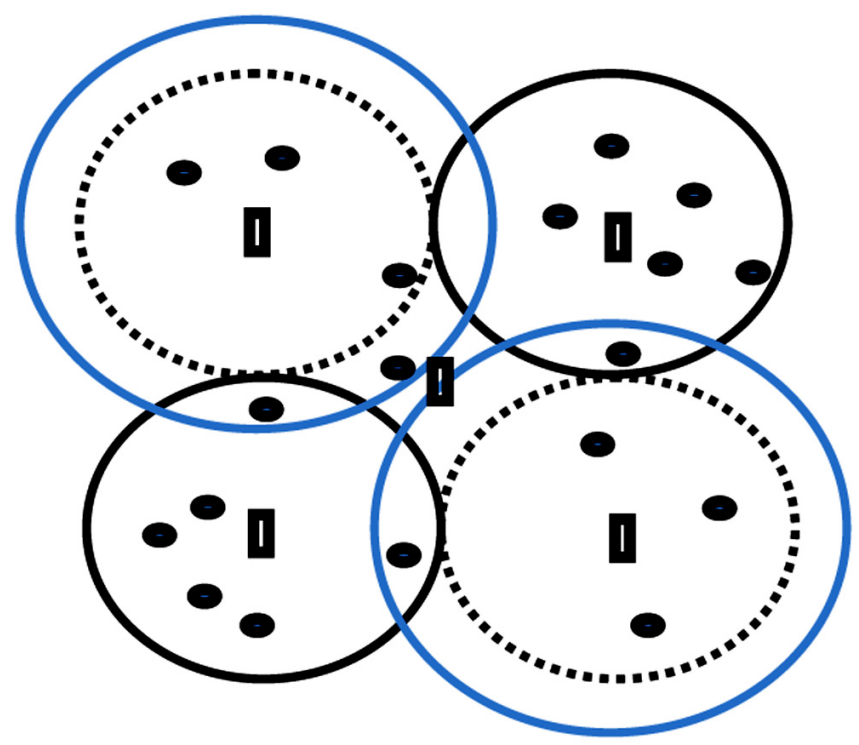

Figure 2 Central cell sleeps and neighboring cell zoom out

to zoom a small distance to give coverage for the users in the switched off cell. Even in this condition only the transmit power of the neighboring BSs is being increased, but still switching off one base station completely saves energy efficiently. This is mainly because the number of base stations are deployed based on the peak traffic and most of the times they are not so.

The changes in BS transmit power before and after cell zooming is analyzed. Similarly, the variation in overall network power consumption before and after switching off cell5 is calculated. The adjustment in transmit power of the MS antenna when it is covered by neighboring cell is considered to be negligible, since it is done in micro cellular condition.

In this scenario the neighboring BSs are assumed to have free channels for the users in the switched off cell and the geographic region of the cell5 could be covered with extending the transmit power of antennas to a maximum transmit limit of 50\%. The network operations are considered to be done in CDMA system and BSs cooperation is used among BSs for cell zooming conditions [10].

The approach reviewed here can be exploited very proficiently in micro, pico and femto cells. But this technique couldn't be used in macrocell conditions, due the larger coverage area, which makes the MSs to double the power consumption than it is. 


\section{Power Consumption Study}

This subdivision gives analysis of power consumption for the examined case. Initially the received power of MS is calculated with Friis Transmission Formula, assuming both transmitting and receiving antennas parameters to be constant. The total BS transmit power is the sum of all the transmission power of BS to each of the users present within the cell. Then the change in BS transmission power before and after cell zooming is compared. The overall power consumption of the network is obtained. Additionally the loss components and interferences are accounted to bring out the system more tangible.

Since receiver power of MS is required to maintain the SNR it is initially calculated. The receiver power of $\mathrm{MS}, \mathrm{P}_{r}$ is calculated by,

$$
P_{r}=\frac{\lambda^{2}}{(4 \pi d)^{2}} P_{t} G_{t} G_{r}
$$

Where, transmitting antenna ( $\mathrm{Tx})$ gain and receiver antenna $(\mathrm{Rx})$ gain is given by $\mathrm{G}_{t} \& \mathrm{G}_{r}$, transmit power is $\mathrm{P}_{t}$, distance from $\mathrm{Tx}$ to $\mathrm{Rx}$ is $d$, also wavelength is given by $\lambda$ respectively.

In this scenario, users are uniformly random distributed in all the five microcells. Since the some of the users in center cell are already in overlapping region and remains users could be certainly covered by neighboring BS with cell zooming out, cell5 is switched off. In order to decide which user to be given coverage among the neighboring cells, optimal solution should be found. Meanwhile the user near to cell1 cannot be covered by the far most cell3, it will cause wastage to power in the cell3. Primarily the distance between users in cell5 and all other cells are calculated and sorted. The nearest neighbor cell zooms out to cover the users in cell5.

Since the users have to receive the same receiver power $\mathrm{P}_{r}$, so as to maintain the minimum SNR, transmit power of the base station has to increase when cell zooms out. The change in transmit power is calculated with the above Eq.1, with the change in the distance value.

Let the transmit power of the BS be $\mathrm{P}_{t}$, the number of users be $\mathrm{K}_{u}, \mathrm{~N}_{b}$ being the number of BSs in the network that is used to cover the users before cell zooming. The total transmit power of the BS before cell zooming $\mathrm{P}_{t x-b z}$ is given by,

$$
P_{t x-b z}=P_{t} \cdot K_{u}
$$

The total transmission power of $\mathrm{BS}$ after cell zooming out $\mathrm{P}_{t x-a z}$, includes the users $\mathrm{E}_{u}$ in cell5. Also the transmission power $\mathrm{P}_{t}$ changes as $\mathrm{P}_{t-\text { mod }}$ due 
the increased distance $\mathrm{d}$ between MS and BS. Thus we find the total transmission power as,

$$
P_{t x-A z}=P_{t-\bmod }\left(E_{u}+K_{u}\right)
$$

The total network power consumption $\mathrm{P}_{n e t}$ includes the power consumption of the total BS present in the network. Where power consumption of BS is the sum of total BS transmission power and $\mathrm{P}_{\text {fixed }}$ which is the stable power consumption of BS that includes the power required for signal processing and power resources used for running the BSs [11]. Thus we get the total network power consumption before cell zooming as,

$$
P_{n e t-B z}=\left(P_{t x-B z}, L_{t}+P_{\text {fixed }}\right) \cdot N_{b}
$$

Where, $\mathrm{L}_{t}$ denotes the total losses due to fading, penetration, shadowing losses of the signal. Similarly, the total network power consumption after cell zooming as,

$$
P_{n e t-A z}=\left(P_{t x-A z} \cdot L_{t}+P_{\text {fixed }}\right) \cdot N_{b}
$$

The difference between the total network power consumption before and after cell zooming gives the amount power saved with cell zooming.

\section{Results and Discussions}

The scenario is examined using MATLAB simulator. The typical constant values used for simulation purpose is listed below in the table I. Also users in the network are spread in a uniform random distribution.

Simulation results for presenting the cellular base station with original size and random distribution of nodes is shown in Fig. 3. Five circles each of radius $200 \mathrm{~m}$ in graph indicate five base stations and the central small circle indicates the center base station (cell5). Each base station coverage users are differentiated by different colours.

Fig. 3 shows that some of the center cell (cell5) users are in edges of circles. Also the other users are easily coverable by extending the coverage of neighbor cells.

Here the neighboring cells are considered to have enough channels for the users of cell5. Thereby the center cell can be switched off. According to the geographic region of the users, the particular users are covered by the closest neighboring cell. This cell zooming out by the neighboring BSs is shown in Fig. 4. 


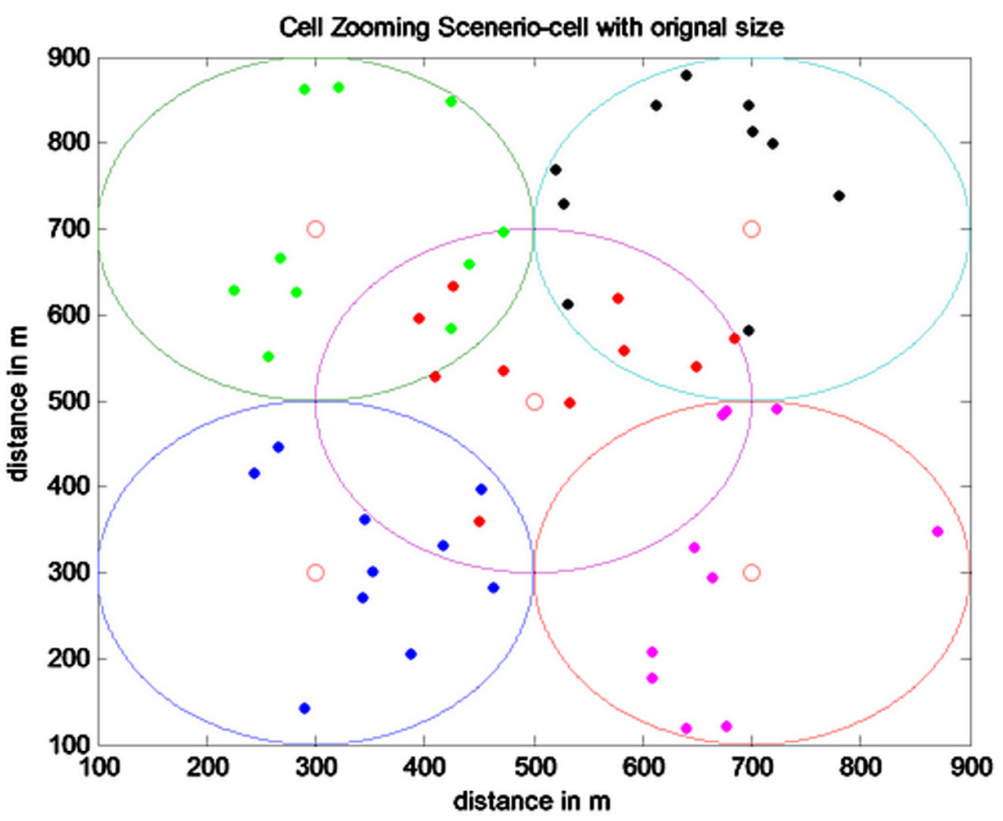

Figure 3 Cell with original size

The reason behind zooming cell 2 and cell 3 is because they are the nearest BSs to the users in the switched off cell, that can be observation from the Fig. 4. Now the transmission power of base station and total network power consumption are analyzed.

The power consumption in general for a BS with respect to the no. of users and distance is presented in Fig. 5. Since the distance and the number of users increase, the transmitted power of BS is also increased. It can be observed that accommodating more users with less power can be possible with smaller cell

Table 1 Analysis parameters

\begin{tabular}{ll}
\hline Parameter & Values \\
\hline Cell radius, $\mathrm{d}$ & $200 \mathrm{~m}, 300 \mathrm{~m}$ (max. when zooms out) \\
Frequency & $1900 \mathrm{MHz}$ \\
Total loss in signal Tx, $\mathrm{L}_{t}$ & $20 \sim 22 \mathrm{~dB}$ \\
MS antenna gain & $-1 \sim 2 \mathrm{~dB}$ \\
BS antenna gain & $0 \sim 2 \mathrm{~dB}$ \\
Fixed power consumption, $\mathrm{P}_{\text {fixed }}$ & $48 \mathrm{dBm}$ \\
\hline
\end{tabular}




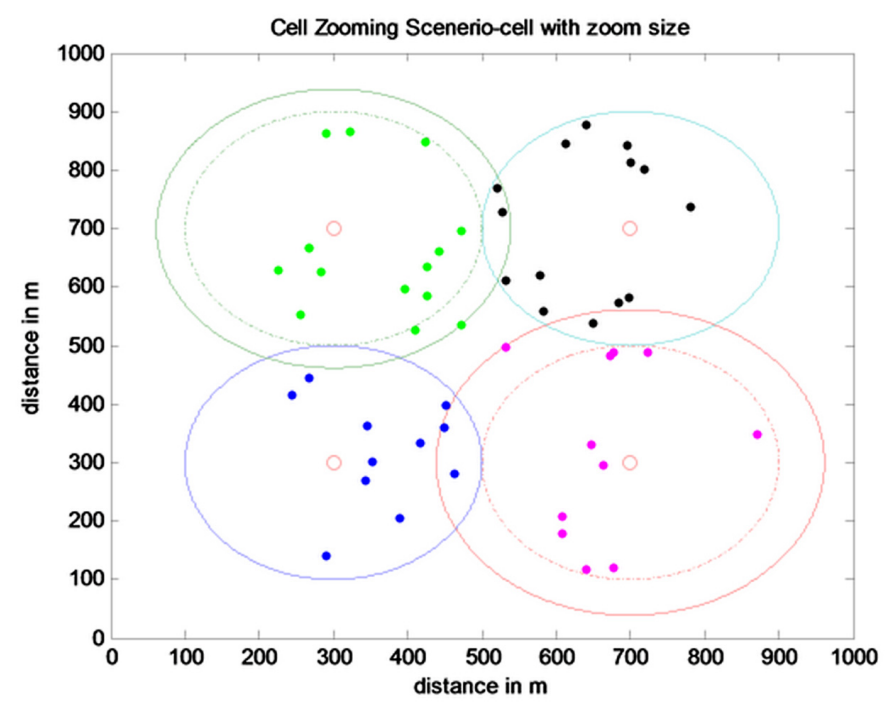

Figure 4 Central cell sleeps and neighboring cell zooms out

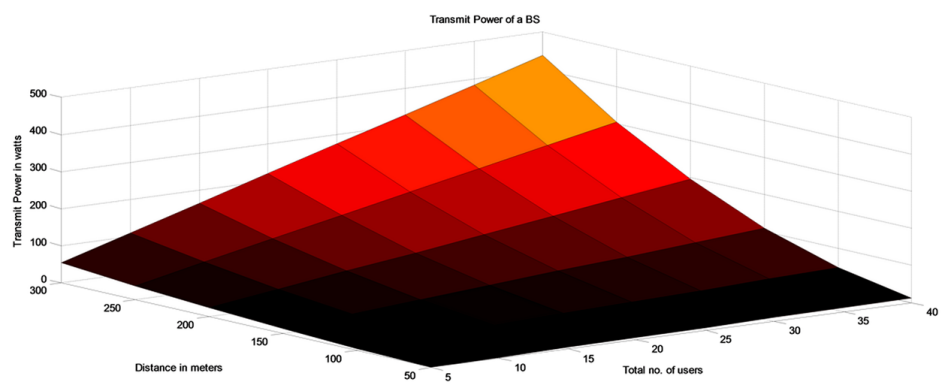

Figure 5 BS transmission power with respect to the no. of users and distance

radius. Thereby, when the traffic is very high, the BSs can zoom in and cover the users nearby the BS with less transmit power.

Fig. 6 shows the BS transmitted power for both the normal coverage $(200 \mathrm{~m})$ and extended coverage with the maximum distance ( $\max$ of $\mathrm{dm}$, i.e., $300 \mathrm{~m}$ ). It can be observed that the BS transmission power is initially differed by only few watts, and the differences exponentially increase with increase in distance. The transmit power of BS is almost double for the distance $\mathrm{dm}$ when comparing with distance $\mathrm{d}$ for 50 users. This is because it covers more users with larger distance. 


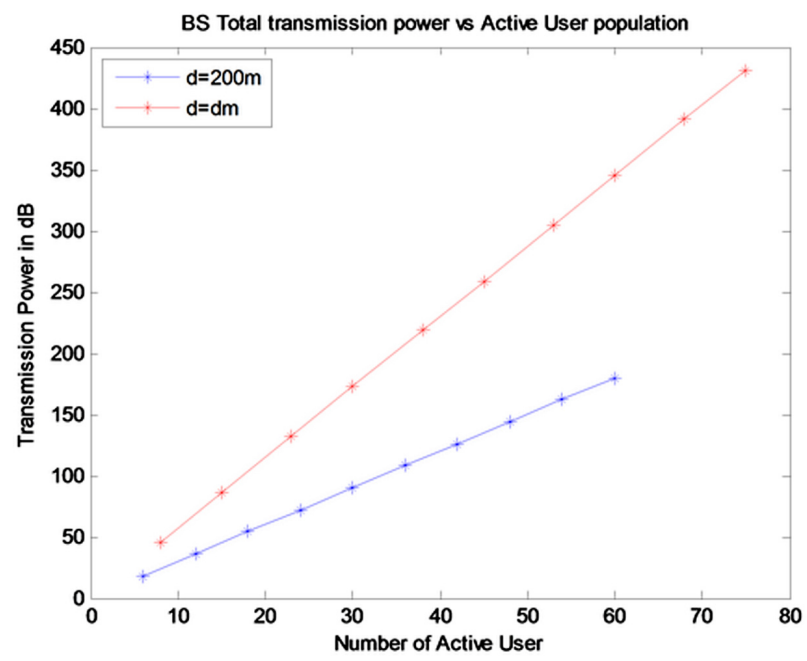

Figure 6 Base station transmission power vs Active user

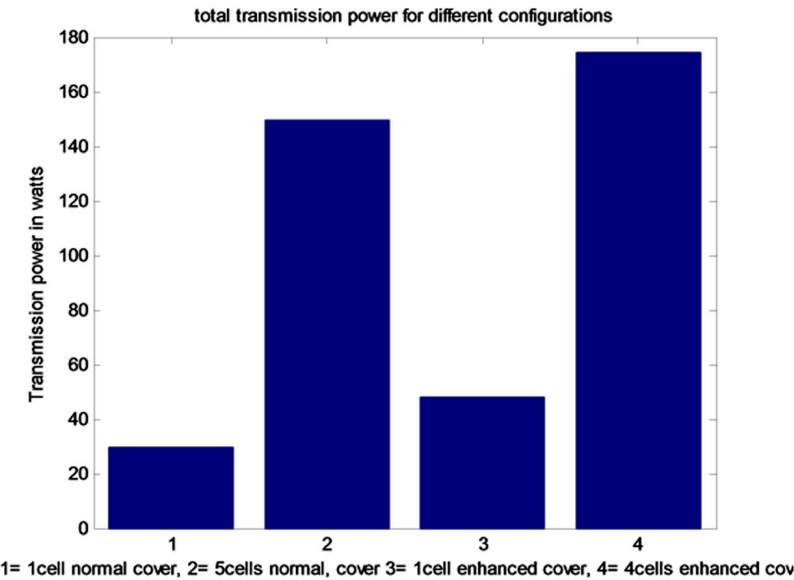

Figure 7 Total BS transmission power for different network configuration

The total BS transmit power of our scenario, is shown in Fig. 7. By comparing the bars one and three it explains that there is a difference of around 20 watts between one cell normal coverage and one cell with enhanced coverage. Also, comparing the bars two and four cells, the transmit power of BS four cells with enhanced coverage consumes 20 watts more than the transmit power of five BS with normal coverage. It should be noted that the transmit power in 


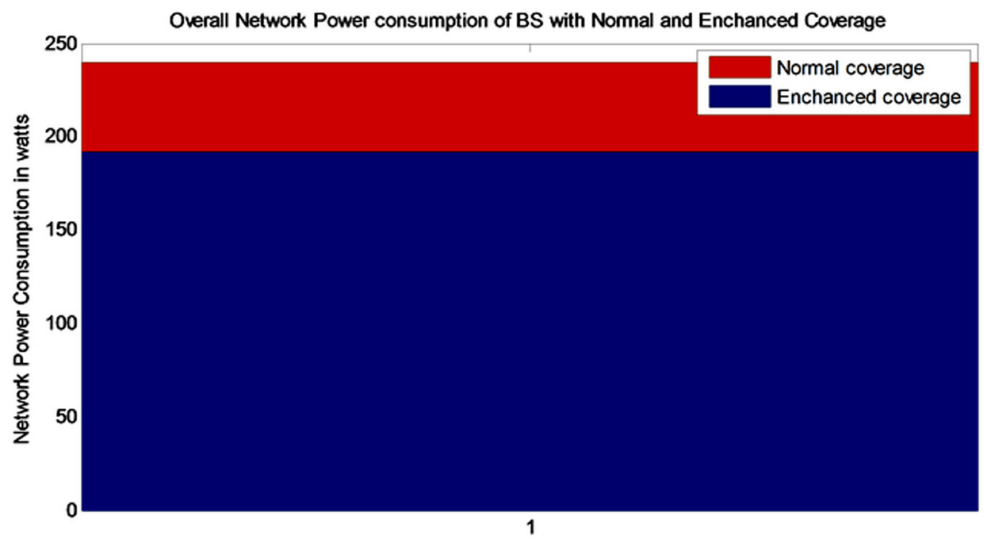

Figure 8 Total network power consumption

bar four is high because it covers more users with larger distance. It clearly explains that the increase in transmit power of BS is there, now the effect of network power consumption has to analyzed for this condition.

We get the total BS power consumption by summing the actual BS transmission power with the stable power consumption of BS. By comparing the bar in Fig. 8, it shows that the network consumes less power with enhanced coverage. It is because that the enhanced coverage is from four BSs with two cells enhancing the coverage while one BS is switchedoff, that saving about 48 Watts in total consumed power. Hence, $20 \%$ of the overall network power is saved with cell zooming concept for the investigated scenario.

\section{Conclusions}

In this paper, an approach is analyzed for reducing the overall network power consumption in a microcellular network by cell zooming technique. Switching off one middle cell when the traffic is less and covering those remaining users by cell zooming out the neighboring base stations in a simple five-cell network configuration is done. The power consumption reduction in the network due to cell zooming is simulated. Results show that the network can save $20 \%$ of the total network power consumption, which is a step towards the implementation of 'green cellular networks'. 


\section{References}

[1] D. Willkomm, S. Manchiraju, J. Bolot and A. Wolisz, "Primary user behavior in cellular networks and implications for dynamic spectrum access", IEEE Commun. Mag., vol. 47, no. 3, pp. 88-95, Mar. 2009.

[2] K. Son, Song Chong and de VecianaG, "Dynamic association for load balancing and interference avoidance in multi-cell networks", IEEE Trans. Wireless Commun., vol. 8, no. 7, pp. 3566-76, July 2009.

[3] White paper - Green Radio "NEC's approach towards energy-efficient radio access networks”, NEC Corporation, Feb. 2010.

[4] L. Chiaraviglio, M.AjmoneMarsan, D.Ciullo and M. Meo, "Energy-Aware UMTS access networks”, Proc. 11th Int'l.Symp. Wireless Pers. Multimedia Commun., Sept. 2008.

[5] M. AjmoneMarsan, L. Chiaraviglio, D.Ciullo and M. Meo, "Energy-Efficient management of UMTS access networks",W-GREEN 2008, Lapland.

[6] M. AjmoneMarsan,L. Chiaraviglio, D. Ciullo and M. Meo "Optimal energy savings in cellular access networks,” IEEE ICC, GreenComWksp., Dresden, Germany, June 2009, pp.1-5, 14-18 june 2009.

[7] X. Weng, Dongxu Cao and Z. Niu, "Energy-efficient cellular network planning under insufficient cell zooming”, in Proc. 73rd Vehic. Technol. Conf., Budapest, Hungary, pp. 1-5, 2011.

[8] Z. Hasan and Vijay K Bhargava, "Green cellular networkA survey, some research issues and challenges”, IEEE Commun. Surveys \& Tutorials, vol. 13, pp. 1-16, Sept. 2011.

[9] Z. Niu, Yanqun Wu, J. Gong and Z. Yang, "Cell zooming for cost-efficient green cellular networks”, IEEE Commun. Mag., vol. 48, no. 11, pp. 74-79, Nov. 2010.

[10] W. C. Y. Lee, "Overview of Cellular CDMA", IEEE Trans. on Vehicular Technology, Vol. 40, No. 2, May 1991.

[11] ZiaulHaNq Abbas and Frank Y. Li, "A system-level power saving approach for cellular networks with microcells/picocells", 2nd International Conference on wireless communication IEEE 2011, Chennai, Mar 3, 2011.

\section{Biographies}

Venkatapathy Prithiviraj Dr. V. Prithviraj is now the principal of Rajalakshmi Institute of Technology, Chennai. He had served from August 2008January 2013 as the Principal of Pondicherry Engineering College. He completed his Bachelor of Engineering (Electronics and Communication Engineering) in 1972 from the College of Engineering, Guindy Madras University, his M.S. by research in 1982 from IIT, Madras, and his Ph.D in 1999 from IIT, Kharagpur (research area - Signal Processing Techniques in Array Antennas Systems).

He is one of the founding members of Pondicherry Engineering College and served the institute from July 1985-2013. He was holding the position of Dean-in-charge, School of Engineering, Pondicherry University from May 
2009-January 2013. He has been teaching for over 30 years and held the position of Head of the Electronics and Communications Engineering Department at PEC from June 2003-2006. He has published over 80 technical research papers.

He has also held the position of Director, IT for Government of Pondicherry (2002-2005). Currently, he is a Member of Expert Committee for monitoring International Indo-French projects in the field of Information Technology as well as Regional Committee of AICTE for Tamil Nadu and Pondicherry. He has a keen interest in research and development projects and provided leadership in many successful projects sponsored by various organizations such as DRDO, ISRO, Department of Electronics and Department of Information Technology at IITs and PEC.

$\mathrm{He}$ is the recipient of the IEEE International Student Branch Award in 1984 and the EDI Award for best technical paper, entitled "COFDM for Telemedicine Applications", in 2007. He is a Life Member of ISTE and Member of EMC Engineers and IEEE USA. His areas of interest include Broad band and Wireless Communication, Mobile Computing, VLSI for Wireless Applications, Tele Medicine, SDR, Cognitive Radio and e-Governance Applications.

S. B. Venkatraman received his M.Tech degree in Wireless Communication Engineering from Pondicherry Engineering College affiliated to Pondicherry University, Pondicherry, India in May 2013. His areas of interests include communication systems, Green Mobile Communications in Cellular Networks, Cognitive Radio and Networking.

R. Vijayasarathi received his M.Tech degree in Wireless Communication Engineering from Pondicherry Engineering College affiliated to Pondicherry University, India in May 2013. His areas of interests include cellular mobile communication, Cognitive Radio, embedded systems and computer networks. 
